\title{
ASSESSMENT OF JAW BONE DENSITY IN TERMS OF HOUNSFIELD UNITS USING CONE BEAM COMPUTED TOMOGRAPHY FOR DENTAL IMPLANT TREATMENT PLANNING
}

\author{
Myra Ahmed, Yasir Ikram*, Farheen Qureshi**, Muhammad Sharjeel***, Zubair Ahmed Khan**, \\ Khurram Ataullah** \\ University College of Dentistry, The University of Lahore, Lahore Pakistan, *21 Military Dental Corps, Quetta/ National University of Medical \\ Sciences (NUMS) Pakistan, **Fatima Memorial Hospital Lahore Pakistan, ${ }^{* * *}$ Armed Forces Institute of Dentistry/National University of \\ Medical Sciences (NUMS) Rawalpindi Pakistan
}

\begin{abstract}
Objective: To assess jawbone density in terms of Hounsfield units using cone beam computed tomography for dental implant treatment planning in patients reporting to a local tertiary care dental hospital

Study Design: Cross sectional study.

Place and Duration of Study: Department of Periodontology and Oral Implantology, Fatima Memorial Hospital, Lahore, from Mar to Sep 2018.

Methodology: A total of 100 patients who fulfilled the inclusion criteria and underwent implant placement were included in the study. After ethical approval, informed and written consent, brief history was taken and a single radiographer exposed and took cone beam computed tomography scan of all the subjects using PLANMECA machine. A single investigator using PLANMECA software recorded jawbone density in terms of Hounsfield units. All data were presented as mean, SD and one way ANOVA was used. Multiple comparisons of the four regions in the maxilla and mandible were performed with a Tukey test. An independent t-test was also used to compare gender with age groups and bone density.

Results: Total of 100 patients who underwent implant placement were included, 48 (48\%) were males \& 52 (52\%) were females with the mean age of $28.53 \pm 5.33$ years. The mean jawbone density in terms of Hounsfield units using cone beam computed tomography in anterior maxilla was $709.75 \pm 122.63$ Hounsfield units, posterior maxilla was $299.66 \pm 73.09$ Hounsfield units, anterior mandible was $1093.34 \pm 109.42$ Hounsfield units and posterior mandible was $599.45 \pm 135.55$ Hounsfield units $(p<.001)$.

Conclusion: The anterior mandible and anterior maxilla has the maximum mean bone density value for the jaw followed by the posterior mandible and the minimum mean bone density value for the posterior maxilla.
\end{abstract}

Keywords: Cone beam CT, Dental implant treatment planning, Hounsfield unit, Jawbone density.

This is an Open Access article distributed under the terms of the Creative Commons Attribution License (http://creativecommons.org/licenses/by/4.0), which permits unrestricted use, distribution, and reproduction in any medium, provided the original work is properly cited.

\section{INTRODUCTION}

Dental implants placement nowadays has become an indispensable part of clinical practice for rehabilitation of partially and totally edentulous patients. Osseointegration at primary and secondary level underlines the success of dental implants ${ }^{1}$. The local bone quantity and quality, surgical technique of placement (i.e., relation between implant size and drill size, whether a pretapped or self-tapped implant is used) and implant geometry, both micro- and macroscopic dictates the primary stability of dental implants ${ }^{2}$. Bone

Correspondence: Dr Yasir Ikram, Flat-D, Block E9, Sarwar Colony, Quetta Cantt Pakistan

Received: 26 Feb 2020; revised received: 30 Mar 2020; accepted: 27 Apr 2020 quality is determined by several factors that contribute to bone strength. However, as an objective measure, physicians use bone density to distinguish the different qualities of the bone. Bone density is an important prerequisite both in the initial implant stabilization and in the loading profile of the prosthesis. Low initial stability and higher implant failure rates in poor quality bone are well known, as compared to higher quality bone $^{3}$. Notably, in the posterior maxilla usually there is the lowest bone quality in the oral setting4.

The correlation between stability of the implants and bone quality suggests that clinicians can predict primary stability before implant place- 
ment and could involve some modifications in their treatment plan, either during the execution of procedure or prior to it (selection of implant, implant position, healing period). Therefore a good preoperative evaluation of bone density can direct the decision of the surgeon regarding patient selection, implant surface and surgical protocol to be used ${ }^{1}$.

Cone-beam computed tomography (CBCT) technology has evolved exponentially in terms of scientific and technological growth with a strong impact in implant dentistry and is currently the most widely used tool for assessing bone quality in both jaws during dental implant planning. The precision of CBCT for determining bone density has also been assessed in different studies,4-6. In this context, Parsa et al 7 , compared CBCT with multislice CT (MSCT) and micro-computed tomography (micro-CT), their results indicated a high association between MSCT and CBCT, demonstrating that $\mathrm{CBCT}$ could be used at the implant site for determining bone mineral density. Although CBCT does not have the accuracy of traditional CT for bone density assessment, it has replaced multislice computed tomography (MSCT) due to advantages offered like greater accuracy, lower radiation exposure, higher resolution, short duration and lower costs 1 .

For CT scans, Hounsfield unit is proportional to the degree of $x$-ray attenuation assigned to each pixel to display the image reflecting the tissue density. The gray scale (voxel value) in CBCT indicates the degree of $x$-ray attenuation, although manufacturers of $\mathrm{CBCT}$ and software suppliers present gray scales as the $\mathrm{HU}^{8}$.

Bone mineral density as CT values (Hounsfield units) was originally categorized by Misch into five ranges to evaluate bone density with D1 being the highest having value $>1,250 \mathrm{HU}$; followed by D2: 850 to 1,250 HU; D3: 350 to $850 \mathrm{HU}$, lowest bone density value of D4: 150 to $350 \mathrm{HU}$ and D5: < $150 \mathrm{HU}$. Additionally, it was observed that the D1 is predominantly present in the anterior mandible, followed by D2 and D3 bone quality in the anterior maxilla and posterior mandi- ble. D4 bone quality is primarily observed in posterior maxilla9.

This study was conducted to assess bone quality at the implant-receiving site prior to implant placement using CBCT as no local study could be found in the literature search. The bone density in local Pakistani population is different from Caucasian and Chinese Asians therefore the mean values of the bone quality formulated in those studies may not serve as references for Pakistani population ${ }^{10}$. The mean values of the bone density prior to surgery shall help the clinicians in treatment planning and will provide recommendations to the clinician in decision regarding the type of implant and surgical protocol.

\section{METHODOLOGY}

The study design was cross sectional and was conducted at Department of Periodontology and Oral Implantology, from Mar to Sep 2018 with permission of Institutional Ethical Review Board (IERB) FMH-06-2018-IRB-469-M.

Both male and female patients with chronological age between 18-40 years indicated to undergo dental implant placement as dictated by his/her treatment plan were considered. Inclusion criteria included medically fit patients, with no history of radiotherapy, chemotherapy, any bone abnormalities in the upper or lower jaw, any orthodontic treatment history, or a CBCT scan in the past 6 months. A sample of 100 was calculated at 5\% level of significance and $p 0.01$ and taking expected mean jawbone density of posterior maxilla as $229.62 \pm 144.48^{8}$. According to Hao et $a l^{8}$, the mean bone density of anterior mandible was greater than anterior maxilla, which was $679.6 \pm 141.67$ and $460.25 \pm 136.42$ respectively. Furthermore, bone density of posterior mandible was reported higher (394.41 \pm 128.37 ) $\mathrm{HU}$ as compared to posterior maxilla ${ }^{8}$. Non-probability consecutive was the sampling technique.

Pregnant or lactating mothers, patients on medications e.g bisphosphonates, with active periodontal disease, retained primary teeth at the site or having any condition affecting bone turnover or bone quality such as osteoporosis were 
excluded. Smoker and alcohol users were also excluded.

All patients who fell into sample selection criteria signed the informed consent form. Demographic details were noted and a thorough dental checkup was carried out. A single radiographer exposed and took CBCT scan (voxel size 200 $\mu \mathrm{m}$, patient dose $\sim 86 \mu \mathrm{Sv}$ ) of all the subjects using PLANMECA (ProMax ${ }^{\circledR}$ 3D Classic, Helsinki, Finland) machine. All scans were viewed in PLANMECA software (Planmeca Romexis software 5.3, Helsinki, Finland) and by using 3D implant verification tool, virtual implant site was planned and on cross-sectional images, was divided into 2 regions: from distal surface of canine into anterior and posterior region. On each image the bone density was measured twice at each implant site. In edentulous patients or partially dentate patients with multiple sites, a mean value was taken to assign for that region. All the information was recorded in a specifically designed proforma. In order to address any bias, all readings were taken by one investigator and CBCT from only one system was included in the study.

Data was entered and analyzed in SPSS version 20. Descriptive statistics were calculated. For qualitative variable like gender, frequency

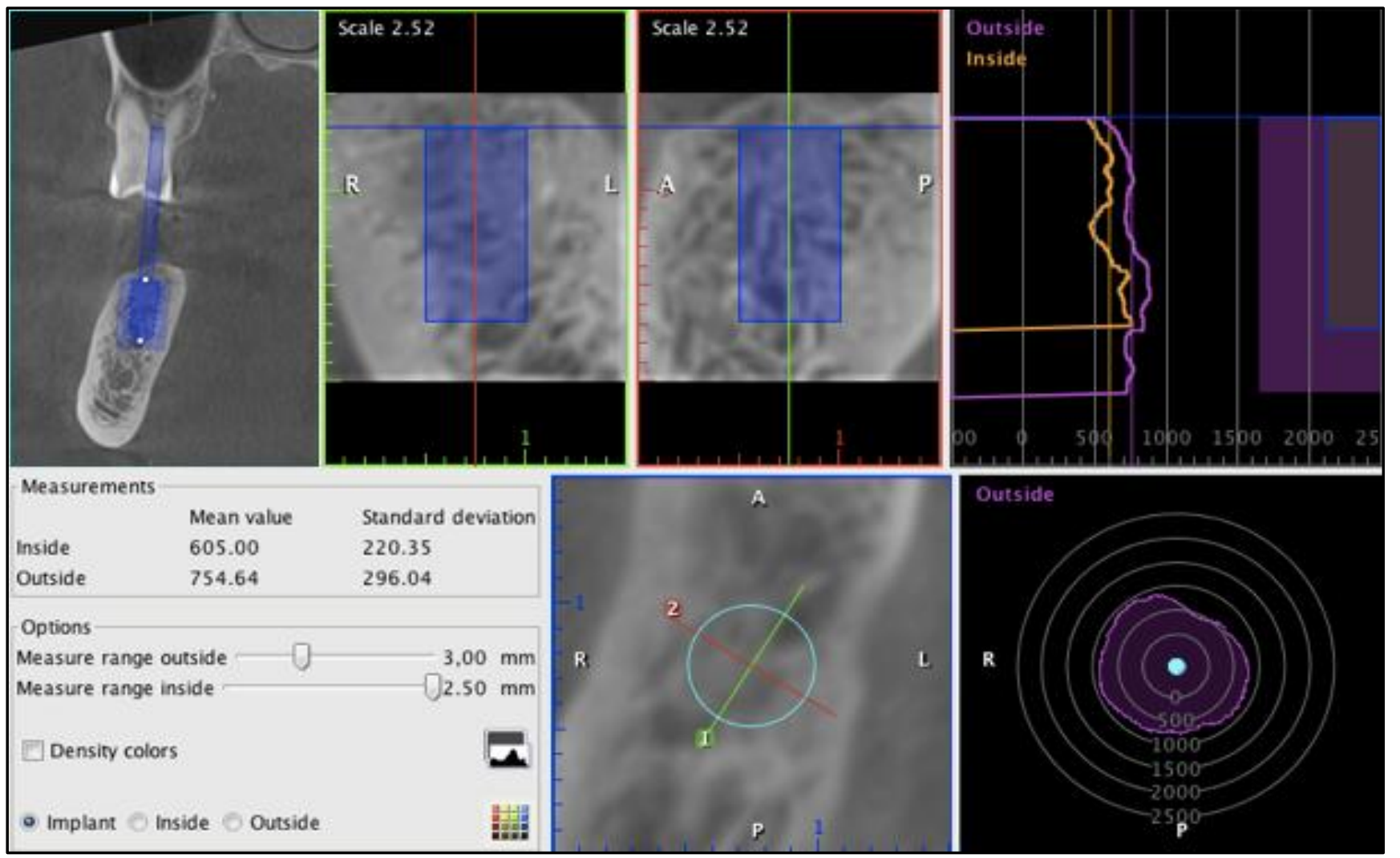

Figure: Virtual implant site with mean bone density values in hounsfield units.

virtually adjusted according to future prosthetic plan. The virtual site of implant was chosen to allow for $2 \mathrm{~mm}$ of distance from the maxillary sinus floor, nasal floor and inferior alveolar canal. Bone density in terms of hounsfields unit was measured at around $1 \mathrm{~mm}$ of virtual implant using the verification tab in the PLANMECA Romexis viewer program (figure). Both jaws were and percentage was calculated. For quantitative variables like bone density and age, mean \pm standard deviation was calculated. ANOVA test was used to determine the differences in the bone density values (HU) between mandible and maxilla. Post Hoc test, Tukey, was used to explore pair-wise difference between 4 regions. Post-stratification independent sample T-test was used to 
compare results between women and men, older and younger patients, as well as among four quadrants of mouth. A value of $p<0.05$ was considered significant.

\section{RESULTS}

A total of 100 patients (48 male, 52 female; mean age $28.53 \pm 5.33$ ) who underwent implant placement as dictated by his / her treatment plan were selected for this study.

The results of One Way ANOVA revealed that there was a significant difference in bone density with regards to anterior maxilla, anterior mandible, posterior maxilla and posterior mandible $(p<.001)$ (table-I). Mean values of jawbone density in terms of Hounsfield units in anterior maxilla was $709.75 \pm 122.63$, posterior maxilla was $299.66 \pm 73.09$ (lowest), anterior mandible was $1093.34 \pm 109.42$ (highest) and posterior mandible was $599.45 \pm 135.55$.

Furthermore, Post Hoc test, Tukey, was used to explore pair-wise difference between 4 regions, while comparing posterior implant recipient sites

Table-I: Region wise comparison of bone density.

\begin{tabular}{|c|c|c|c|c|c|c|}
\hline & \multirow[b]{2}{*}{ Location } & \multirow{2}{*}{$\begin{array}{c}\text { Mean } \pm \text { Std. } \\
\text { Deviation }\end{array}$} & \multicolumn{2}{|c|}{$95 \%$ Confidence Interval } & \multirow[b]{2}{*}{$\mathbf{F}$} & \multirow[b]{2}{*}{$\begin{array}{c}p \text { - } \\
\text { value }\end{array}$} \\
\hline & & & $\begin{array}{l}\text { Lower } \\
\text { Bound }\end{array}$ & $\begin{array}{l}\text { Upper } \\
\text { Bound }\end{array}$ & & \\
\hline \multirow{4}{*}{$\begin{array}{l}\text { Bone } \\
\text { Density }\end{array}$} & Anterior Maxilla $(\mathrm{n}=100)$ & $709.75 \pm 122.63$ & 685.419 & 734.085 & \multirow{4}{*}{848.436} & \multirow{4}{*}{$<0.001$} \\
\hline & Posterior Maxilla $(\mathrm{n}=100)$ & $299.66 \pm 73.09$ & 285.157 & 314.162 & & \\
\hline & Anterior Mandible $(\mathrm{n}=100)$ & $1093.42 \pm 109.42$ & 1071.63 & 1115.054 & & \\
\hline & Posterior Mandible $(n=100)$ & $599.45 \pm 626.34$ & 572.553 & 626.346 & & \\
\hline
\end{tabular}

Table-II: Pair-wise difference of bone density.

\begin{tabular}{l|c|c|c|c|c}
\hline & $\begin{array}{c}\text { Mean } \\
\text { Difference }\end{array}$ & $\begin{array}{c}\text { Std. } \\
\text { Error }\end{array}$ & \multirow{2}{*}{$\boldsymbol{p}$-value } & \multicolumn{2}{|c}{ 95\% Confidence Interval } \\
\cline { 4 - 7 } & Lower Bound & Upper Bound \\
\hline Anterior Maxilla- Posterior Maxilla & 410.093 & 15.926 & $<0.001$ & 369.003 & 451.182 \\
\hline Posterior Maxilla-Anterior Mandible & -793.683 & 15.926 & $<0.001$ & -834.772 & -752.593 \\
\hline Anterior Mandible-Posterior Mandible & 493.893 & 15.926 & $<0.001$ & 452.803 & 534.982 \\
\hline Posterior Mandible- Anterior Maxilla & -110.303 & 15.926 & $<0.001$ & -151.392 & -69.213 \\
\hline Posterior Maxilla-Posterior Mandible & -299.79 & 15.926 & $<0.001$ & -340.879 & -258.701 \\
\hline Anterior mandible- Anterior Maxilla & 383.59 & 15.926 & $<0.001$ & 342.500 & 424.679 \\
\hline
\end{tabular}

Table-III: Stratification of mean jawbone density in terms of Hounsfield Units using CBCT for dental implant treatments in different anatomic locations with respect to age and gender.

\begin{tabular}{|c|c|c|c|c|c|}
\hline Gender & Bone Density & Age Group & Mean \pm Std. Deviation & t-test & $p$-value \\
\hline \multirow{8}{*}{ Male } & \multirow{2}{*}{$\begin{array}{c}\text { Anterior } \\
\text { Maxilla }\end{array}$} & $18-30(n=30)$ & $722.958 \pm 128.441$ & \multirow{2}{*}{0.918} & \multirow{2}{*}{0.364} \\
\hline & & $31-40(n=18)$ & $687.653 \pm 130.126$ & & \\
\hline & \multirow{2}{*}{$\begin{array}{c}\text { Posterior } \\
\text { Maxilla }\end{array}$} & $18-30(n=30)$ & $294.842 \pm 80.405$ & \multirow{2}{*}{-0.626} & \multirow{2}{*}{0.535} \\
\hline & & $31-40(n=18)$ & $308.667 \pm 61.934$ & & \\
\hline & \multirow{2}{*}{$\begin{array}{c}\text { Anterior } \\
\text { Mandible }\end{array}$} & $18-30(n=30)$ & $1095.108 \pm 118.530$ & \multirow{2}{*}{1.06} & \multirow{2}{*}{0.295} \\
\hline & & $31-40(n=18)$ & $1057.875 \pm 116.510$ & & \\
\hline & \multirow{2}{*}{$\begin{array}{l}\text { Posterior } \\
\text { Mandible }\end{array}$} & $18-30(n=30)$ & $593.467 \pm 150.417$ & \multirow{2}{*}{-0.052} & \multirow{2}{*}{0.959} \\
\hline & & $31-40(n=18)$ & $595.708 \pm 137.129$ & & \\
\hline \multirow{8}{*}{ Female } & \multirow{2}{*}{$\begin{array}{c}\text { Anterior } \\
\text { Maxilla }\end{array}$} & $18-30(n=31)$ & $674.234 \pm 116.936$ & \multirow{2}{*}{-2.816} & \multirow{2}{*}{0.007} \\
\hline & & $31-40(n=21)$ & $762.262 \pm 100.337$ & & \\
\hline & \multirow{2}{*}{$\begin{array}{c}\text { Posterior } \\
\text { Maxilla }\end{array}$} & $18-30(n=31)$ & $302.919 \pm 68.392$ & \multirow{2}{*}{0.427} & \multirow{2}{*}{0.672} \\
\hline & & $31-40(n=21)$ & $294.012 \pm 81.457$ & & \\
\hline & \multirow{2}{*}{$\begin{array}{l}\text { Anterior } \\
\text { Mandible }\end{array}$} & $18-30(n=31)$ & $1122.944 \pm 98.263$ & \multirow{2}{*}{1.62} & \multirow{2}{*}{0.112} \\
\hline & & $31-40(n=21)$ & $1077.524 \pm 100.590$ & & \\
\hline & \multirow{2}{*}{$\begin{array}{l}\text { Posterior } \\
\text { Mandible }\end{array}$} & $18-30(n=31)$ & $594.258 \pm 122.261$ & \multirow{2}{*}{-0.675} & \multirow{2}{*}{0.503} \\
\hline & & $31-40(n=21)$ & $618.869 \pm 138.699$ & & \\
\hline
\end{tabular}


as compared to anterior in each jaw, higher bone density was found in anterior regions which was highly significant $(p<0.001)$ (table-II). Whereas while comparing, anterior mandibular region with posterior maxilla a significant difference in mean bone density was noted (MD=-793.68, $p<0.001)$. In addition to this, the mean difference between posterior mandible and anterior maxilla was found to be significant (MD=-110.303, $p<0.001)$. The negative mean difference showed that anterior maxillary bone density was more as compared to posterior mandibular bone density. While comparing posterior maxillary bone density with posterior mandibular bone density, the significant difference was found (MD=-299.79, $p<0.001)$ which showed that posterior mandibular density was high as compared to posterior maxillary bone density. Lastly, the mean difference between anterior mandible and anterior maxilla was made and significant results were obtained ( $\mathrm{MD}=383.59, p<0.001)$. The results showed that bone density is higher at anterior mandible as compared to anterior maxilla.

Stratification of mean jawbone density in terms of Hounsfield Units using CBCT for dental implant treatment in different anatomic locations with respect to age groups and gender was calculated. The results of independent sample t-test (table-III) revealed that no significant difference was found between the two age groups in males with regards to bone density in anterior maxilla $(\mathrm{t}=0.918, p=0.364)$, posterior maxilla $(\mathrm{t}=0.626, p=$ $0.535)$, anterior mandible $(\mathrm{t}=1.06, p=0.295)$ and posterior mandible $(t=0.052, p=0.959)$. While in females, the results revealed that significant difference was found between the two age groups with regards to bone density in anterior maxilla $(t=-2.816, p=0.007)$ while no significant difference was found between the two age groups with regards to bone density in posterior maxilla $(t=.427$, $p=0.672)$, anterior mandible $(\mathrm{t}=1.62, p=0.112)$ and posterior mandible $(\mathrm{t}=-0.675, p=0.503)$.

\section{DISCUSSION}

Precise and objective pre-operative assessment of bone density at future implant site pro- vides pivotal recommendations to the clinicians on dental implant treatment planning. Radi IA ${ }^{4}$ reported failures that may be associated with low bone quality values in groups. Therefore, a robust quantitative scale range, rather than absolute values, can provide flexible and precise facilitation to the clinician in order to classify bone quality making it easier to decide when to load an implant immediate, early, or delayed.

Many published methods are suggested for bone quality assessment, but most of them showed a lack of objectivity since they rely on the practitioner and/or can only be used during (osteotomy preparation) or after surgery (subsequent to implant placement) ${ }^{11,12}$. This study used the protocol applied in preceding research to assess bone densities at multiple sites and reported bone density measurements using CBCT as HU. While Misch's classification and Lekholm and Zarb's classification was based on the HU created from $\mathrm{CT}$, the positive high correlation between the $\mathrm{HU}$ derived from $\mathrm{CBCT}$ and $\mathrm{CT}$ is still well documented ${ }^{13}$.

Computed tomography (CT) determination of the bone anatomy and density is more precise. In addition, it was also concluded that CBCT generated $\mathrm{HU}$ value as compared to $\mathrm{CT}$ for the same bone area was higher ${ }^{13}$. However, CBCT and CT generated density values have been found to be similar to the Lekholm and Zarb classification based bone density values with correlation coefficient reported to be $0.59-0.61^{14}$.

D1-D4 (Lekholm \& Zarb) bone densities were found throughout all the edentulous sites in this study, with significant differences $(p<0.001)$ in bone densities in the four areas of mouth, the highest mean bone density values in anterior mandible, followed by the anterior maxilla, posterior mandible and the lowest mean bone density values for posterior maxilla, similar to the values reported in the study by Hao et al ${ }^{8}$. Furthermore, the mean bone density was 299.66 $\pm 73.09 \mathrm{HU}$ in this study, when considering all implant sites in posterior maxilla, which was lower than that stated by Norton and Gamble $463.7 \pm 290 \mathrm{HU}^{9}$. 
In this study the mean jawbone density in terms of Hounsfield Units using CBCT for dental implant treatments in anterior maxilla was $709.7525 \pm 122.634 \mathrm{HU}$, posterior maxilla was $299.66 \pm 73.090 \mathrm{HU}$, anterior mandible was $1093.3425 \pm 109.424 \mathrm{HU}$ and posterior mandible was $599.4500 \pm 135.552 \mathrm{HU}$, as compare to Turkyilmaz et al15. Where mean bone density values were higher $721 \pm 291,505 \pm 274 \mathrm{HU}$ in posterior mandible, posterior maxilla and lower $927 \pm 237$, $708 \pm 277$, in anterior mandible and anterior maxilla respectively as compared to this study. Furthermore, the mean bone density at the maxillary posterior region $299.66 \pm 73.090 \mathrm{HU}$ was lower than in other reported studies $\mathrm{UK}^{9}(417.3$ $\pm 227.3 \mathrm{HU})$, USA16 (333 $\pm 199 \mathrm{HU})$ and Saudi Arabia $^{17}$ (320.05 $\left.\pm 193.6 \mathrm{HU}\right)$. With the exception of D1 in the posterior maxilla and D4 in the anterior mandible, D1 to D4 were present in all the regions analyzed. The type 4 bone needs a thorough surgical technique execution and clinicians need careful planning in the posterior maxilla for implant size, number of implants and progressive loading. Moreover, most bone in all implant sites has been classified as D2 and D3 (74.15 percent), which is adequate for implantation ${ }^{15}$.

In the anterior mandible, anterior maxilla, posterior mandible, and posterior maxilla, the mean bone density value is lower than those previously reported 15,18 ; the discrepancy may result from the differences in CBCT and CT scanners. While other factors could be the differences in patient's age, bone mineral density and gender, which have been, reported in previous studies 15,18 .

A strong correlation was found between the bone sites subjectively classified as D1 or D4 (based on resistance to drilling), and the histomorphometrically measured bone density as stated in the previous study of Trisi et al19. However, the subtle differences between D2 and D3 could not be differentiated in this way.

A close relationship has been reported in patients with poor bone density having increased implant failure rates, Jaffin and Berman ${ }^{20}$ recor- ded $3 \%$ for type 1,2 , and 3 bone, but $35 \%$ for type 4 bone, as classified by Lekholm and Zarb. Because of implant failure this type D4 bone requires meticulous surgical technique.

Type D1, with bone density (1093.3425 \pm 109.424 HU) has an increased risk of overheating during installation of the dental implants. According to the lowest tolerance limit of D1, the values above $\pm 600 \mathrm{HU}$ indicate bone with increased density. D2 and D3 were combined into one group, the differentiation difficulties between D2 and D3 have been previously reported in other studies based on a subjective visual assessment or quantitative bone density measurement ${ }^{21,22}$. Between $\mathrm{HU} \pm 200$ and $\mathrm{HU} \pm 600$ intermediate values (D2/3) are suitable conditions for osseo-integration.

Some previous studies $2,22,23$ reported that intensity values in CBCT sections is not reliable because image parameters and positioning affected the values. Nevertheless, the results of this study indicate a high degree of concordance between the different regions of the oral cavity and the different bone densities, with a correlation between the four qualities.

\section{CONCLUSION}

The anterior mandible has the highest mean jawbone density $(1093.3425 \pm 109.424$ HU) followed by anterior maxilla, posterior mandible and the lowest mean jawbone density of the posterior maxilla (299.66 $\pm 73.090 \mathrm{HU})$. Therefore, it is suggested that an objective assessment supporting the value of a site-specific examination of bone density prior to installation of the implants can provide valuable information to the clinicians placing implants in our population so that they might amend their selection of implant size, drilling protocol and treatment duration. In addition, protocols for immediate loading developed using research on different populations must be implemented with caution.

\section{CONFLICT OF INTEREST}

This study has no conflict of interest to be declare by any author. 


\section{REFERENCES}

1. Rios HF, Borgnakke WS, Benavides E. The use of cone-beam computed tomography in management of patients requiring dental implants: an american academy of periodontology best evidence review. J Periodontol 2017; 88(10): 946-59.

2. Liu J, Chen HY, DoDo H, Yousef H, Firestone AR, Chaudhry J, et al. Efficacy of cone-beam computed tomography in evaluating bone quality for optimum implant treatment planning. Implant Dent 2017; 26(3): 405-11.

3. Javed F, Ahmed HB, Crespi R, Romanos GE. Role of primary stability for successful osseointegration of dental implants: Factors of influence and evaluation. Interv Med Appl Sci 2013; 5(4): 162-67.

4. Radi IA, Ibrahim W, Iskandar SMS, Nabi AN. Prognosis of dental implants in patients with low bone density: A systematic review and meta-analysis. J Prosthetic Denistr 2018; 120(5): 668-77.

5. Pauwels R. Cone beam CT for dental and maxillofacial imaging: Dose matters. RadiatProt Dosimetry 2015; 165(1-4): 156-16.

6. Felicori MS, Da Gama SR, Silvaet QC. Assessment of maxillary bone density by the tomodensitometric scale in Cone-Beam Computed Tomography (CBCT). J Health Sci Inst 2015; 33(4): 319-22.

7. Parsa A, Ibrahim N, Hassan B, Motroni A, P van der Stelt, Wismeijer D. Influence of cone beam CT scanning parameters on grey value measurements at an implant site. Dentomaxillofac Radiol 2013; 42(3): 79884780.

8. Hao Y, Zhao W, Wang Y, Yu J, Zou D. Assessments of jaw bone density at implant sites using 3D cone-beam computed tomography. Eur Rev Med Pharmacol Sci 2014; 18(9): 1398-403.

9. Norton MR, Gamble C. Bone classification: an objective scale of bone density using the computerized tomography scan. Clin Oral Implants Res 2001; 12(1): 79-84.

10. Khan AH, Jafri L, Ahmed S. Osteoporosis and its perspective in Pakistan: A review of evidence and issues for addressing fragility fractures. Ann Med Surg (Lond) 2018; 29(1): 19-25.

11. Bruno V, Berti C, Barausse C, Badino M, Gasparro R, Ippolito DR, Felice P. Clinical relevance of bone density values from CT related to dental implant stability: A retrospective study. Bio- med Res Int 2018; 2018: 6758245.

12. Guerra ENS, Almeida FT, Bezerra FV, Figueiredo PTDS, Silva MAG, De Luca Canto G, et al. Capability of CBCT to identify patients with low bone mineral density: a systematic review. Dentomaxillofac Radiol 2017; 46(8): 20160475.

13. Aranyarachkul P, Caruso J, Gantes B, Schulz E, Riggs M, Dus I, et al. Bone density assessments of dental implant sites: 2. Quantitative cone-beam computerized tomography. Int J Oral Maxillofac Implants 2005; 20(3): 416-24.

14. Lee S, Gantes B, Riggs M, Crigger M. Bone density assessments of dental implant sites: 3 . Bone quality evaluation during osteotomy and implant placement. Int J Oral Maxillofac Implants 2007; 22(2): 208-12.

15. Turkyilmaz I, TozumTF, Tumer C. Bone density assessments of oral implant sites using computerized tomography. J Oral Rehabilitation 2007; 34(4): 267-72.

16. Shapurian T, Damoulis PD, Reiser GM, Griffin TJ, Rand WM. Quantitative evaluation of bone density using the Hounsfield index. Int J Oral Maxillofac Implants 2006; 21(2): 290-97.

17. Almasoud NN, Tanneru N, Marei HF. Alveolar bone density and its clinical implication in the placement of dental implants and orthodontic mini-implants.Saudi Med J. 2016; 37(6): 684-89.

18. Pauwels R, Jacobs R, Singer SR, Mupparapu M. CBCT-based bone quality assessment: are Hounsfield units applicable? Dentomaxillofac Radiol 2015; 44(1): 20140238.

19. Trisi P, Rao W. Bone classification: clinical-histomorphometric comparison. Clin Oral Implants Res 1999; 10(1): 1-7.

20. Jaffin RA, Berman CL. The excessive loss of Branemark fixtures in type IV bone: a 5-year analysis. J Periodontol 1991; 62(1): 2-4.

21. Lekholm U, Zarb Ga. Patient selection and preparation In: Brånemark PI, Zarb GA, Albrektsson Ts. Tissue integrated prostheses: osseointegration in clinical dentistry. Chicago: Quintessence Publishing Co. 1985; 199-209.

22. Nackaerts O, Maes F, Yan H, Souza CP, Pauwels R. Analysis of intensity variability in multislice and cone beam computed tomography. Clin Oral Implants Res 2011; 22(8): 873-79.

23. Wada M, Tsuiki Y, Suganami T, Ikebe K, Sogo M. The relationship between the bone characters obtained by CBCT and primary stability of the implants. Intl J Implant Dent 2015; 3(1): 1-5. 